\title{
Epigenetic variation taking center stage in immunological research
}

\author{
"Among the main goals of BLUEPRINT was to \\ improve our knowledge about natural variation \\ between and within individuals."
}

First draft submitted: 20 January 2017; Accepted for publication: 20 January 2017; Published online: 21 March 2017

Keywords: autoimmune disease $\bullet$ cell differentiation $\bullet$ chromatin $\bullet$ DNA methylation - epigenetics $\bullet$ hematopoiesis $\bullet$ heterogeneity $\bullet$ human immune system $\bullet$ leukemia $\bullet$ natural variation

\section{The BLUEPRINT epigenome project}

The recent publication of a collection of 41 papers by the International Human Epigenome Consortium (IHEC) [1] has shed exciting new light on epigenomes and epigenetic mechanisms in health and disease $[2,3]$. The majority of these publications focused on different aspects of immune biology which was the main topic of BLUEPRINT [4], the European contribution to IHEC. BLUEPRINT generated over 100 complete and over 1000 partial reference epigenomes of healthy and diseased blood cells [5,6], and tackled many functional and mechanistic questions [2]. Here, we highlight six studies of the collection addressing three questions fundamental to the wider research community with interest in immunity and epigenomics: what is the level of natural epigenetic variation in blood cells? What is the effect of the microenvironment or residency on the epigenome of blood cells? What is the cell type of origin in hematological malignancies?

Every nucleated cell in a multicellular organism contains an almost identical copy of the genetic code, except for somatic mutations. Yet cells differ dramatically in shape, size and function. Distinct cell types can develop because of the synthesis of different RNAs in time and space, and therefore different protein molecules. This is the most fundamental level at which the genotype gives rise to the phenotype, forming the basic principle for cellular differentiation and the versatility and adaptability of any cell and, in particular, of immune cells.

Beside genetic recombination used by $\mathrm{T}$ and $\mathrm{B}$ cells to generate a diverse repertoire of cell surface receptors and immunoglobulins, respectively, immune responses are driven by key signaling elements and the control of transcription factors [7] which are both subject to epigenetic modulation. Epigenetic modifications are changes on top of the genetic material which mark the genome by the use of chemical moieties, leading to alterations in the regulatory and transcriptional potential of a cell. Cellular epigenetic states are highly dynamic and contribute to phenotypic plasticity, enabling flexible and rapid responses to external cues [7].

The importance of epigenetic regulation in response to infection and inflammation is well established [7,8]. Other environmental factors such as nutrition, stress or exercise also induce changes in the epigenome. Thus, the epigenome forms the intersection of the genome and the environment, and is therefore crucial to the understanding of the human immune system and its function in health and disease.

Among the main goals of BLUEPRINT was to improve our knowledge about natural variation between and within individuals. The comprehension of variation in physiology is essential to understand human disease and to translate this vital research into the clinic for

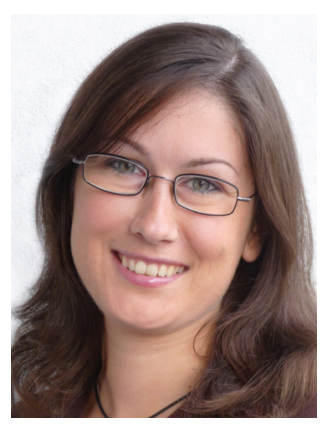

Simone Ecker Author for correspondence: UCL Cancer Institute, University College London, 72 Huntley Street, London WC1E 6BT, UK s.ecker@ucl.ac.uk

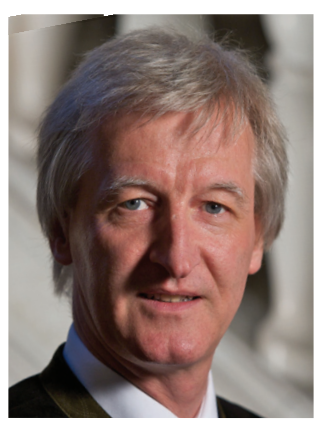

Stephan Beck

UCL Cancer Institute, University College London, 72 Huntley Street, London WC1E 6BT, UK 
the ultimate benefit to patients. So far, interindividual and intraindividual variability have mainly been studied at the genetic level, particularly in human cancer. BLUEPRINT successfully demonstrated the advantage of using epigenomic data to provide a better understanding of the development of immune-related diseases, and to identify their cell types of origin. For many immunological malignancies, the cell type giving rise to the disorder is still unknown. Its correct identification is crucial for the development of therapeutic agents targeting the corresponding cell type. Multiple studies of the BLUEPRINT consortium demonstrated that epigenomes reflect the cellular identity in the hematopoietic tree allowing us to identify which hematopoietic cell type gave rise to which hematopoietic malignancy. Adding further complexity, however, epigenetic variation is also present across individual cells within a given cell type. For example, changes in the microenvironment have been demonstrated to alter the epigenetic patterns of blood cells, highlighting the importance of the interaction between the epigenome and the environment.

\section{Natural epigenetic variation in human immune cells}

Study 1 tackled the important question of normal epigenetic variation at the population level by generating high-resolution datasets of human primary $\mathrm{CD} 14^{+}$ monocytes, $\mathrm{CD} 16^{+}$neutrophils and $\mathrm{CD} 4^{+} \mathrm{T}$ cells from around 200 individuals [9]. To investigate the chain of steps in between DNA sequence and protein variation, genomes, epigenomes and transcriptomes of all three cell types were derived from this set of individuals. Associations to transcription factor binding, chromatin states, alternative splicing and protein abundance showed highly coordinated genetic effects in all three cell types. Despite cis-genetic effects explaining the major part of transcriptomic variability, over 2000 genes were found to be associated with epigenetic variation independent of genetics. These genes with purely epigenetic associations were particularly enriched in immunological pathways, further highlighting the importance of epigenetic effects in the human immune system. In addition, identified molecular quantitative trait loci (QTLs) co-localized with previously reported loci associated with autoimmune disease.

"Changes in the microenvironment have been demonstrated to alter the epigenetic patterns of blood cells, highlighting the importance of the interaction between the epigenome and the environment."

Study 2 investigated the promoter interactome in 17 human hematopoietic cell types by $\mathrm{Hi}-\mathrm{C}$, showing that interactions were enriched for links between promoters and enhancers, and that active enhancers had an additive effect on cell type-specific gene expression [10]. Additionally, promoter-interacting regions were selectively enriched for expression QTLs regulating the same gene, providing exciting further insights into the regulatory mechanisms of genome-epigenome interactions. QTLs were also identified at promoterinteracting regions which have effects on more than one gene. Finally, genetic variants associated with diseases were shown to be significantly enriched at promoter-interacting regions, again emphasizing the link between genetic variation and epigenetic interactions in human disease.

\section{Epigenetic variation in hematopoiesis \& the microenvironment}

Studies 3-5 provided new interesting insights into the epigenetic dynamics of human hematopoiesis by demonstrating progressive epigenetic changes in blood cell maturation, particularly at the level of DNA methylation [11-13].

Employing a metaepigenomic approach, study 3 used a combination of pooled and single-cell methylome sequencing to establish genome-wide reference maps of DNA methylation dynamics in human hematopoiesis [11]. The work demonstrated that DNA methylation profiles can be used to predict cell type throughout the human hematopoietic lineage with high accuracy, and emphasized the progressive epigenetic remodeling occurring during human blood cell maturation. DNA methylation in hematopoietic stem cells derived from fetal liver, bone marrow and blood was shown to be different. Furthermore, the innate and adaptive human immune systems exhibit substantially distinct DNA methylation dynamics. Through the analysis of 112 whole-genome bisulfite sequencing datasets, study 4 showed DNA methylation patterns to be quite stable during differentiation in the myeloid lineage, but demonstrated a pronounced progressive loss of DNA methylation in lymphocyte development [12]. Consistent with these results, study 5 reported global loss of DNA methylation during T memory cell differentiation following profiling of human $\mathrm{CD}^{+} \mathrm{T}$ cells [13]. Interestingly, it was shown that tissue-resident bone marrow $\mathrm{T}$ memory cells have a distinct epigenetic profile compared with their circulating counterparts, further highlighting the importance of taking the microenvironment into account.

\section{Epigenetic variation in hematologic malignancies}

It is well known that the epigenome is strongly altered in disease. However, the question of if and how these epigenetic modifications drive disease development 
remains elusive. Analyzing blood cancer cells, study 6 showed that an epigenetic imprint reflecting the type and differentiation stage of the cell of origin is present in malignant cells [14]. The identification of the epigenetic 'memory' of these cells not only helps to gain insight into the pathogenesis of immune-related diseases, but also enables patient stratification into novel clinical disease subtypes. The analysis of 82 DNA methylation signatures of mantle cell lymphomas, a subtype of B-cell lymphoma, identified two new subgroups of the disease. Cells of these two subtypes reflect imprints of germinal center-inexperienced and germinal center-experienced B cells, showing that DNA methylation profiles during lymphomagenesis are influenced by methylation dynamics of normal B-cell development. Similar findings had been previously reported for chronic lymphocytic leukemia [15,16], another blood cancer affecting $\mathrm{B}$ cells. This leukemia shows only few genetic alterations, but extensive epigenomic deregulation which has been associated with clinical characteristics and patient subgroups $[15,17,18]$.

\section{Conclusion \& future perspective}

Taken together, the study of epigenomes of normal cell types is indispensable to improve our understanding of the epigenetic basis of human hematopoiesis. It is well known that alterations of the epigenome outside development and differentiation affect cellular function and contribute to disease development. Epigenomes of normal cell types provide valuable information about human physiology. In comparison to their diseased counterparts, they contribute to our understanding of the mechanisms involved during disease development, and can reveal the particular cell types driving immune-related diseases and their subtypes, which are still often unknown. A deep under-

\section{References}

Papers of special note have been highlighted as: $\bullet$ of interest

1 International Human Epigenome Consortium. http://ihec-epigenomes.org

2 Stunnenberg HG, The International Human Epigenome Consortium, Hirst M. The International Human Epigenome Consortium: a blueprint for scientific collaboration and discovery. Cell 167(5), 1145-1149 (2016).

3 Cell Press. Insights from the International Human Epigenome Consortium (2016).

http://www.cell.com/consortium/IHEC

4 BLUEPRINT Epigenome. http://www.blueprint-epigenome.eu

5 Bujold D, Morais DA de L, Gauthier C et al. The International Human Epigenome Consortium Data Portal. Cell Syst. 3(5), 496-499 (2016). standing of the heterogeneity present in the human immune system in both health and disease will be key to enhance the development of therapeutic approaches for immune-related diseases. The data and findings presented in the IHEC collection open promising new avenues for basic and clinical research into immune and other diseases.

\section{"A deep understanding of the heterogeneity present in the human immune system in both health and disease will be key to enhance the development of therapeutic approaches for immune-related diseases."}

Despite the enormous progress already made, much remains to be done to translate the new knowledge gained from the integration of multidimensional omics data into the clinic, particularly for other common diseases in addition to cancer. One of the many efforts promising to address this is MultipleMS [19], a new H2020 Consortium of $21 \mathrm{EU}$ and US partners. MultipleMS will develop, validate and exploit multiomic data together with lifestyle and clinical information for patient stratification in multiple sclerosis, a chronic inflammatory disease.

\section{Financial \& competing interests disclosure}

Support for this work was provided by the UK National Institute for Health Research UCLH Biomedical Research Center (BRC84/CN/SB/5984) and the H2020 Project MultipleMS (733161). The authors have no other relevant affiliations or financial involvement with any organization or entity with a financial interest in or financial conflict with the subject matter or materials discussed in the manuscript apart from those disclosed.

No writing assistance was utilized in the production of this manuscript.

6 Fernández JM, de la Torre V, Richardson D et al. The BLUEPRINT Data Analysis Portal. Cell Syst. 3(5), 491-495 (2016).

7 Busslinger M, Tarakhovsky A. Epigenetic control of immunity. Cold Spring Harb. Perspect. Biol. 6(6), a019307 (2014).

8 Obata Y, Furusawa Y, Hase K. Epigenetic modifications of the immune system in health and disease. Immunol. Cell Biol. 93(3), 226-232 (2015).

9 Chen L, Ge B, Casale FP et al. Genetic drivers of epigenetic and transcriptional variation in human immune cells. Cell 167(5), 1398-1414 (2016).

- Study of genome, epigenome and transcriptome variation in human monocytes, neutrophils and $\mathrm{T}$ cells identifying the contribution of genetics and epigenetics to transcription.

10 Javierre BM, Burren OS, Wilder SP et al. Lineage-specific genome architecture links enhancers and non-coding disease 
variants to target gene promoters. Cell 167(5), 1369-1384 (2016).

- High-resolution maps of promoter interactions in 17 human primary blood cell types showing that promoterinteracting regions are enriched for regulatory features and expression quantitative trait loci, and associated with disease.

11 Farlik M, Halbritter F, Müller F et al. DNA methylation dynamics of human hematopoietic stem cell differentiation. Cell Stem Cell 19(6), 808-822 (2016).

- DNA methylation maps of hematopoietic stem and progenitor cells demonstrating that methylation differs between the myeloid and lymphoid lineages, and in different microenvironments.

12 Schuyler RP, Merkel A, Raineri E et al. Distinct trends of DNA methylation patterning in the innate and adaptive immune systems. Cell Rep. 17(8), 2101-2111 (2016).

- Analyzing trends of DNA methylation in the myeloid and lymphoid lineage, and reporting progressive loss of methylation during lymphocyte development.

13 Durek P, Nordström K, Gasparoni G et al. Epigenomic profiling of human $\mathrm{CD}^{+} \mathrm{T}$ cells supports a linear differentiation model and highlights molecular regulators of memory development. Immunity 45(5), 1148-1161 (2016).

- Epigenomic analysis of human $\mathrm{CD}^{+}{ }^{+} \mathrm{T}$ memory cell subsets proposing a linear model of memory development in circulating $\mathrm{T}$ cells, and showing that tissue-resident bone marrow $\mathrm{T}$ memory cells exhibit a different epigenetic profile.

14 Queirós AC, Beekman R, Vilarrasa-Blasi R et al. Decoding the DNA methylome of mantle cell lymphoma in the light of the entire B cell lineage. Cancer Cell 30 (5), 806-821 (2016).

- Revealing two clinically different subtypes of mantle cell lymphoma characterized by epigenetic imprints of distinct normal B-cell subpopulations.

15 Kulis M, Heath S, Bibikova $M$ et al. Epigenomic analysis detects widespread gene-body DNA hypomethylation in chronic lymphocytic leukemia. Nat. Genet. 44(11), 1236-1242 (2012)

16 Kulis M, Heath S, Castellano G et al. Whole-genome fingerprint of the DNA methylome during human B-cell differentiation. Nat. Genet. 47(7), 746-756 (2015).

17 Rendeiro AF, Schmidl C, Strefford JC et al. Chromatin accessibility maps of chronic lymphocytic leukaemia identify subtype-specific epigenome signatures and transcription regulatory networks. Nat. Commun. 7, 11938 (2016).

18 Queirós AC, Villamor N, Clot G et al. A B-cell epigenetic signature defines three biological subgroups of chronic lymphocytic leukemia with clinical impact. Leukemia 29(3), 598-605 (2014).

19 Community Research and Development Information Service. MultipleMS (2016).

http://www.multiplems.eu 